\section{RÖNTGEN CENTENARY AND FIFTY YEARS OF X-RAYS}

\author{
By Prof. J. A. CROWTHER \\ University of Reading
}

$\mathrm{W}$

ILHELM KONRAD RONTGEN was born on March 27, 1845. The discovery of $\mathrm{X}$-rays was communicated by him to the Physico-Medical Society of Würtzburg in November 1895; a translation of his paper appeared in Nature of January 23, 1896. The present year thus marks the centenary of the birth of the discoverer, and the fiftieth anniversary of the epoch-making discovery. with which his name is associated. It has sometimes been suggested that the discovery of $\mathrm{X}$-rays was a happy accident; but there is no doubt, from the nature of his preparations, that Röntgen had, as he himself stated, set out to see whether the electric discharge through a gas at low pressure gave out any kind of 'invisible radiation' capable of detection outside the walls of the glass tube in which the discharge was taking place. The discharge tube (an ordinary Crookes tube of the cylindrical pattern, with a flat cathode at one end, and an anode tucked away in a side tube) had been wrapped in black paper, to cut off all the visible glow from the discharge, and a primitive fluorescent screen, consisting of a few crystals of barium platinocyanide on a piece of cardboard, lay handy on an adjacent bench-barium platinocyanide being a substance commonly used at the time to detect the invisible rays in the solar spectrum. On exciting the tube by means of a small induction coil to see if the light from the discharge was properly obscured by its black paper wrapper, Röntgen found that this was, indeed, the case; but he also noticed that his primitive fluorescent screen was now glowing brightly. The discovery of X-radiation had been made.

It was as simple as that; but it was not 'accidental', unless it be regarded as a happy accident that Röntgen should have thought it worth while to investigate a possibility so remote from current physical thought that, although research into the physics of the Crookes tube was being actively pursued in some of the leading physical laboratories, no other experimenter had thought it worth while to make the very simple tests involved. Some workers had, in fact, been inconvenienced by the fogging of photographic material kept in the same laboratory as their Crookes tubes; but, finding that the trouble could easily be overcome by storing the material elsewhere, they proceeded with the researches on which they were engaged without further thought or hindrance.

Röntgen showed that the new X-radiation affected a photographic plate, and he was able to take a photograph through the thickness of a stout pinewood door separating two of his laboratories. He also noticed that a strip of beading, which had been stuck on the door panel with white lead, cast a distinct shadow on the negative; the thin layer of lead being much more opaque to the radiation than the wood itself. Thus the foundations of the science of radiography were laid.

Both for its practical importance, and for its immense value as a tool for further research, the discovery of $\mathrm{X}$-rays ranks high among the germinal discoveries of physics. The power of the radiation to penetrate considerable thicknesses of materials opaque to ordinary light, and to cast shadows of structures of greater or smaller density concealed within them, caused considerable popular excitement, and had obvious possibilities. The fact that the discovery was first communicated to a medical society has been taken as an indication that Röntgen immediately foresaw its importance in medical diagnosis; though the possibility that he merely took the readiest means of establishing his claim to what was clearly a discovery of first importance cannot altogether be overlooked. However this may be, the new radiation was taken up by members of the medical profession in all countries, in Britain among the first, with a zeal and devotion quite beyond praise. The pioneers among them began work with apparatus little more effective than, and quite as troublesome as, that with which Röntgen made the original discovery. A radiograph of even such an easy subject as the human hand required an exposure measured in minutes. Gradually, however, the insistent demands of the medical radiologists for more and more penetrating power, and shorter and shorter exposures, bore fruit. With the realization that there would be a substantial market for X-ray equipment, engineers became interested in the problem, and the modern diagnostic set is capable of producing excellent radiographs of any part of the human body in a matter of seconds, and with little more trouble to the operator than would be involved in switching on an ordinary electric lamp.

Progress in the interpretation of the radiographs has been equally marked. In medicine, $\mathrm{X}$-rays have evolved from an exciting novelty into a standard routine. Some of the pioneers are with us still; others, we recall, as in duty bound, lost their lives through their devotion to their work, since the dangerous nature of the radiation was not at first realized.

It is not the purpose of this brief article to sketch the story of the evolution of medical radiography, but its recent invasion of the field of preventive and social medicine deserves at least a passing notice. Mass radiography, that is to say, the routine radiography of all workers, has already begun, and will undoubtedly be extended as conditions permit. In such diseases as pulmonary tuberculosis, early diagnosis is nine-tenths of the battle, and the presence of the disease can often be identified by radiography long before any physical symptoms occur to direct attention to it. In a recent survey of some 20,000 operatives, some $1 \frac{1}{2}$ per cent were found to be affected, and were sent for treatment. Thus some three hundred workers will owe their escape from serious incapacity, and possibly from a premature death, to this development of Röntgen's discovery of X-rays.

The use of X-rays in the treatment of disease has scarcely made such satisfactory progress as its use in diagnosis. Many malignant growths, sarcomas, carcinomas and the like respond rapidly to the action of radiation, and fade away under its influence as if by magic. For many types of these scourges upon humanity, X-radiation, either alone or accompanied by radium radiation (which, after all, is only a kind of naturally occurring X-radiation), is by far the most hopeful treatment, and no great hospital is complete without a well-equipped and well-staffed radiotherapy department. It must be confessed, however, that there is as yet an uncertainty about the action of the rays which is very tantalizing. Complete success appears to be so near, and yet continues to elude us. The action of the X-rays on tissue cells, whether healthy or diseased, is, it must be 
understood, always destructive; the possibility of using $\mathrm{X}$-rays to cure disease rests on the fact that malignant cells are, on the whole, less resistant to the action of the rays than healthy tissue. Some of our troubles are undoubtedly due to the difficulty of administering a lethal dose to the malignant growth without damaging the surrounding healthy tissues. This is, primarily, a problem in physics, and the appointment of qualified physicists to the staff of the radiotherapy departments, which is now becoming general, will undoubtedly lead to some solution in due course. Probably a more vital handicap has been that little or nothing was known of the way in which the radiation exerted its destructive powers on the cell. Treatment, therefore, has had to be largely empirical. Recently, however, thanks to ingenious co-operation between physicists and cytologists, it begins to look as if a breach had been made into the problem. If so, not only may methods of treatment gain in certainty, but also X-rays may add a new chapter to our knowledge of the living cell, as they have already added more than one to our knowledge of the atom.

Industry, on the whole, was slower to grasp the utility of X-rays than the medical profession. Some small amount of radiographic testing of materials for hidden defects was carried out during the War of 1914-18, mainly in connexion with aeroplane construction, in which the importance of ensuring the complete soundness of every part justified the very considerable skill and trouble involved in making adiographs with the comparatively primitive apparatus then available. Gradually, however, the value of $\mathrm{X}$-rays as a non-destructive method of investigating the soundness of castings, weldings and other industrial products received fuller recognition, and the number of industrial radiologists, in this second World War, must very greatly exceed that of medical radiologists. The demands of industry, even more effective than those of medicine, since they are backed with much larger capital, have resulted in still greater advances in the design of X-ray apparatus. Millionvolt $X$-ray tubes, enclosed with the high-tension generator which feeds them in a single unit weighing three-quarters of a ton, are now on the market, and in heavy industry (in which they are mainly em. ployed) are regarded as quite portable. With such an outfit, successful radiographs can be made through a 6-in. steel plate, and, under suitable conditions, cracks or flaws of no more than a tenth of an inch in thickness can be detected with certainty.

To one who began X-ray work not many years later than Röntgen's original discovery, the contrast between one of these huge but obedient 'genii of the lamp' and the miscellaneous and extremely temperamental assortment of physical apparatus with which Röntgen first revealed the existence of X-rays (and which, with a few minor improvements, served experimenters for the next twenty years or so) is astounding. By their creation, applied science has gone far to repay the debt which it owes to pure science for the discovery of the radiation. When times are again favourable, pure science will no doubt respond by using these powerful tools to make still fresh discoveries. Nor has the limit yet been reached. A two-million volt tube, some $10 \mathrm{ft}$. long, is now in operation, while another carries (for a millionth of a second) a current of 2,000 amperes at 300,000 volts ; and has been employed for making instantaneous radiographs of the passage of a highvelocity shell through a $1 \frac{1}{2}$-in. armour plate.
Still higher voltages, accompanied by still greater penetration and power in the radiation, is promised by a new type of generator, working on entirely novel principles, which has recently been constructed in the United States. From this machine, which has been christened the 'betatron', X-radiation at energies up to thirty million volts can be obtained. When it is remembered that the most penetrating radiation from radium is equivalent to $\mathrm{X}$-radiation at only two million volts, it will be realized that the betatron carries us into an entirely novel and unexplored region of the X-ray spectrum. Such radiation will penetrate not merely the outworks, but also the central citadel or nucleus of the atom; and its effects, particularly in radiotherapy, are quite unpredictable. They may be beneficial, or disastrous ; but it is evident that we cannot afford to leave them unexplored. It is greatly to be hoped that the various bodies in Great Britain, whether Governmental or voluntary, interested in radiotherapeutics, and particularly in cancer research, will make the necessary provision for British research workers (who have already done so much, often with painfully inadequate resources) to play their part in the quest.

It is natural, in tracing the consequences and developments of Röntgen's discovery, to dwell first on its more immediate applications. X-radiation was, however, one of those germinal discoveries, which are at least as important for the new knowledge which they make possible as for their direct utility. Coming, as it did, a year or so before the complementary discovery of the electron by Sir J. J. Thomson, it opened the way to entirely new regions of experimental research, including the structure of atoms and molecules, and the nature of radiation itself. It may well be that when the final balance comes to be struck, the importance of X-rays as a means of winning fresh knowledge may be judged to outweigh their more directly utilitarian merits.

A knowledge of the nature of the rays was a necessary preliminary to their intelligent application in further research. The true explanation was put forward by Stokes within a few months of Röntgen's discovery. He suggested they were electromagnetic pulses produced by the sudden stoppage of the electrified particles carrying the discharge, and therefore of the same nature as light (though probably of much shorter wave-length). So far, however, did Röntgen's discovery stand apart from the general trend of the physical thought of the time, that there was an interval in which some physicists were inclined to associate the radiation with the vivid fluorescence of the glass walls of the Crookes tube, which is an invariable accompaniment of the electric discharge. Becquerel was led to investigate whether X-rays were also given off by naturally occurring fluorescent materials. Uranium was one of the substances tested, and thus the natural radioactivity of uranium was discovered. The isolation of radium, by the Curies, followed. Thus the whole science of radioactivity may be regarded as a direct offspring, though perhaps an illegitimate one, of Röntgen's discovery.

Although Stokes's suggestion was soon generally accepted, and was supported by Barkla's demonstration that X-rays, like light, could be polarized, progress was at first slow. Such evidence as there was indicated that the wave-length of the radiation must be of the order of one ten-thousandth of that of ordinary light, and attempts to apply the diffraction 
methods used in determining optical wave-lengths seemed almost hopeless. It was not until 1912, when Laue suggested that the regular spacings of the atoms in a crystal might serve as a naturally occurring diffraction grating for X-rays, that real progress was made. The suggestion was immediately verified by Friedrich and Knipping, and in the following year W. L. Bragg, by a most ingenious combination of crystallography and optical theory, succe日ded in determining simultaneously the grating constant of a rock-salt crystal, and the wave-length of the X-radiation employed.

This achievement has a double significance. If the spacing of the atoms in a crystal is known, we can, from diffraction measurements, determine the wavelength of the radiation impinging upon it, and thus obtain an X-ray spectrum. Conversely, if the wavelength of the radiation is known, we can deduce the spacings or lattice constants of the crystal. Thus, in the X-ray spectrometer, crystallography has found a most powerful research tool which has given quite a new impetus to this rather old-fashioned science. The practical importance of X-ray erystallography in metallurgy can scarcely be over-estimated. Since each chemical compound has its own unique set of lattice constants, it is possible to identify, not merely the elements present in a given substance (a task for which ordinary spectroscopy or chemical analysis will suffice), but also the actual compounds which these elements form with each other, and the temperatures at which these compounds form, or at which they disappear. When the field has been fully surveyed, and the $\mathrm{X}$-ray crystallographic index which is "already in active preparation is completed, it will be possible to identify any crystalline compound in an alloy or mixture by X-ray analysis. Nor is the field limited to true crystals. Many long-chain organic substances are sufficiently regular in their make-up to act as X-ray gratings, and much information, valuable both on the theoretical and the industrial side, has already been obtained (to quote only one example) on the structure of the fibres used in the weaving of textiles. To understand why substances behave as they do, in terms of the position of the atoms within them, is the first step in a scientific search for new and better materials; and X-ray crystallography seems destined to play a part in industry even more important than in radiography.

To attempt to survey the part played by X-rays in atomic research would involve the recital of the greater part of the history of that part of the subject dealing with the structure of the electron atmosphere which surrounds the central nucleus and on which most of the chemical and physical properties of the atom depend. Most of our exact knowledge-and it is very exact-of the arrangement, or to be more precise, the energy-levels, of the electrons in the atom, is derived from $\mathrm{X}$-ray data. It may be recalled that Moseley's early survey of the characteristic X-ray spectra given out by the elements provided the first clear demonstration of the fundamental importance of the idea of atomic number; that is to say, the charge upon the atomic nucleus as opposed to the atomic weight, which was shown to be of only secondary importance. It is interesting to notice that Moseley's results showed that four elements still remained to be discovered, and that two of these have since been identified by their X-ray spectra. It may also be mentioned that the most accurate determinations of the two fundamental physical constants, the charge on an electron and Planck's constant, are, in all probability, those derived from $\mathrm{X}$-ray measurements.

The temptation to moralize on the history of the discovery of $\mathbf{X}$-radiation, and its consequences, is strong; but the task may be left to the reader. Nature is full of surprises, and it is not always the most obviously desirable researches which yield the richest harvest. The lone experimenter who is prepared to follow his own inspiration, even if it seems to lead him away from the main current of research, has often played an important part in the progress of science; and pure science in particular, and society in general, owe much to the inspired curiosity of Wilhelm Konrad Röntgen.

\section{DIFFRACTION METHODS IN MODERN STRUCTURAL CHEMISTRY}

$\mathrm{N}$ the Tilden Lecture delivered before the Chemical I Society on January 18, Prof. J. M. Robertson outlined first the scope, limitations and possible future developments of the X-ray and electron diffraction methods, and then went on to consider the nature and lengths of bonds with particular reference to the results of recent diffraction studies. The emphasis throughout was on organic structures, as would be expected from one who has contributed more than any other single worker to our knowledge of the precise crystal structures of organic compounds.

In comparing the electron and X-ray diffraction methods, a number of points were brought out. The great value of the former is that it is applicable to gases and the vapours of easily volatile substances, which cannot conveniently be studied by the X-ray method. In this sense the two methods are complementary. We might add that, as regards inorganic compounds, they are complementary in another sense. In general, the same finite molecules exist in the vapour as in the crystals of an organic compound, but this is not usually so in inorganic chemistry. Electron diffraction studies of the vapours of many metallic salts and of some of the compounds of nonmetals give information about the structures of molecules which do not exist in the crystalline material.

A comparison was made of the nature of the experimental data obtainable by the two methods and of the ways in which they are interpreted. To the eye the electron diffraction photograph shows merely a number of rings on a background of decreasing intensity, and their positions and intensities are estimated visually. The X-ray photograph from a single crystal, on the other hand, shows a large number of discrete spots, and the positions and intensities of these can be determined with consider. able accuracy. This apparent advantage of the X-ray method is, however, offset by two complications. First, the molecules in the gas scatter independently of one another and they are oriented in all possible ways. This makes it possible to calculate the diffraction effects to be expected for any given molecular model and to compare them with those observed, the parameters in the model being varied until agreement is obtained. In the case of diffraction by a crystal, this cannot be done. In the crystal the molecules are definitely oriented with respect to one another, so that not only has the molecular model to be varied 